\title{
AN IMPROVED POINCARÉ INEQUALITY
}

\author{
RITVA HURRI-SYRJÄNEN
}

(Communicated by Palle E. T. Jorgensen)

\begin{abstract}
We show that a large class of domains $D$ in $\mathbb{R}^{n}$ including John domains satisfies the improved Poincaré inequality

$$
\left\|u(x)-u_{D}\right\|_{L^{q}(D)} \leq c\left\|\nabla u(x) d(x, \partial D)^{\delta}\right\|_{L^{p}(D)}
$$

where $p \leq q \leq \frac{n p}{n-p(1-\delta)}, p(1-\delta)<n, \delta \in[0,1], c=c(p, q, \delta, D)<\infty$, and $u$ is in an appropriate Sobolev class.
\end{abstract}

\section{INTRODUCTION}

In this note we improve standard versions of the Poincare inequality. My work was stimulated by a paper of $\mathrm{H}$. Boas and E. Straube [BS]. They showed that a bounded domain whose boundary is locally the graph of a Hölder continuous function of order $\delta, 0 \leq \delta \leq 1$, satisfies the following type of Poincaré inequality:

$$
\left\|u(x)-u_{D}\right\|_{L^{p}(D)} \leq c\left\|\nabla u(x) d(x, \partial D)^{\delta}\right\|_{L^{p}(D)},
$$

where $d(x, \partial D)$ is the distance from $x \in D$ to the boundary of $D, c=$ $c(p, \delta, D)<\infty$, and $u \in L^{p}(D)$ is a function from $W_{p, \text { loc }}^{1}(D)$.

We study the following generalization of (1.1):

$$
\inf _{a \in \mathbb{R}}\|u(x)-a\|_{L^{q}(D)} \leq c\left\|\nabla u(x) d(x, \partial D)^{\delta}\right\|_{L^{p}(D)},
$$

where $p \leq q \leq \frac{n p}{n-p(1-\delta)}$, when $p(1-\delta)<n$, and $c=(p, q, \delta, D)<\infty$. If this inequality (1.2) is true for all $u \in L_{\mathrm{loc}}^{1}(D)$ such that $\nabla u(x) d(x, \partial D)^{\delta} \in$ $L^{p}(D)$, we write $D \in \mathscr{P}(q, p, \delta)$.

This inequality is an improvement of the ordinary $(q, p)$-Poincare inequality when $\delta=0$. There are ordinary $(p, p)$-Poincare domains which do not satisfy the improved Poincare inequality for any $\delta>0$ (see Remark 3.11(4) and $[B S, 4(1)])$. Our main theorems are

Received by the editors May 4, 1992.

1991 Mathematics Subject Classification. Primary 46E35, 26D10.

Key words and phrases. Poincaré inequality, Poincaré domains, John domains, domains satisfying a quasihyperbolic boundary condition.

This paper was written while the author was visiting the University of Texas at Austin. She wishes to thank the Department of Mathematics for its hospitality.

(C) 1993 American Mathematical Society $0002-9939 / 93 \$ 1.00+\$ .25$ per page 
1.3. Theorem. Suppose that $D$ in $\mathbb{R}^{n}$ is a b-John domain, $b \geq 1$. If $D$ is bounded, then $D \in \mathscr{P}(q, p, \delta)$ whenever $p \leq q \leq \frac{n p}{n-p(1-\delta)}, p(1-\delta)<n$, and $\delta \in[0,1]$. If $D$ is unbounded, then $D \in \mathscr{P}(q, p, \delta)$ whenever $p \leq q=$ $\frac{n p}{n-p(1-\delta)}, p(1-\delta)<n$, and $\delta \in[0,1]$.

1.4. Theorem. Suppose that $D$ in $\mathbb{R}^{n}$ satisfies a quasihyperbolic boundary condition with a constant $a$, and let $|D|<\infty$. The domain $D \in \mathscr{P}(q, p, \delta)$ whenever $p \leq q \leq \frac{(n-\lambda) n p}{a(n-p(1-\delta))}$ and $p(1-\delta)<n$; here $\delta \in[0,1)$ and $\lambda<n$ is a Whitney cube \#-constant.

We give the proofs of Theorems 1.3 and 1.4 in $\S 3$. There we show that the bounds for $\delta, p$, and $q$ are essentially sharp. Theorems 1.3 and 1.4 improve results in [BS]. For related background we refer the reader to [EO, H2, K, M] .

\section{Preliminaries}

Notation. Throughout this paper we let $D$ be a domain of euclidean $n$-space $\mathbb{R}^{n}, n \geq 2$. We suppose that $p \in[1, \infty), q \in[1, \infty)$, and $\delta \in[0,1]$ unless otherwise stated.

The space $L^{p}(D)$ is the set of Lebesgue measurable functions $u$ on $D$ for which $\|u\|_{L^{p}(D)}^{p}=\int_{D}|u(x)|^{p} d x<\infty$. Let $L_{\text {loc }}^{p}(D)$ denote the space of functions which are locally integrable of order $p$ on $D$. The space of Lebesgue measurable functions on $D$ with first distributional partial derivatives in $L^{p}(D)$ is denoted by $L_{p}^{1}(D)$. In the Sobolev space $W_{p}^{1}(D)=L^{p}(D) \cap L_{p}^{1}(D)$ we use the norm $\|u\|_{W_{p}^{1}(D)}=\|u\|_{L^{p}(D)}+\|\nabla u\|_{L^{p}(D)}$. Here $\nabla u=\left(\partial_{1} u, \ldots, \partial_{n} u\right)$ is the distributional gradient of $u$. We let $W_{p \text {, loc }}^{1}(D)$ denote the space of functions that lie in $W_{p}^{1}(A)$ for every compact subset $A$ of $D$.

The average of a function $u$ over a domain $D$ with finite Lebesgue measure $|D|$ is $u_{D}=\frac{1}{|D|} \int_{D} u(x) d x$. Let $A$ be a set. The euclidean distance from $x \in A$ to the boundary of $A$ is written as $d(x, \partial A)$. We let $\operatorname{dia}(A)$ denote the diameter of $A$. We write $\tau Q$ for the cube with the same center as $Q$ and dialated by a factor $\tau>1$.

We let $c(*, \ldots, *)$ denote a constant which depends only on the quantities appearing in the parentheses.

$(q, p)$-Poincaré domains. Let $D \subset \mathbb{R}^{n}$ be a domain, and let $1 \leq p \leq q<\infty$. If there is a constant $c=c(p, q, D)<\infty$ such that

$$
\inf _{a \in \mathbb{R}}\|u-a\|_{L^{q}(D)} \leq c\|\nabla u\|_{L^{p}(D)}
$$

whenever $u \in L_{p}^{1}(D)$, then $D$ is a $(q, p)$-Poincaré domain and we write $D \in$ $\mathscr{P}(q, p)$.

John domains. Let $E$ be a closed arc with endpoints $a$ and $b$. The subarc between $x$ and $y$ is denoted by $E[x, y]$. For $x$ in $E \backslash\{a, b\}$ write

$$
q(x)=\min \{\operatorname{dia}(E[a, x]), \operatorname{dia}(E[b, x])\} .
$$

Let $c \geq 1$. A domain $D$ in $\mathbb{R}^{n}$ is a c-John domain, if each pair of distinct points $a$ and $b$ in $D$ can be joined by an arc $E$ such that

$$
\operatorname{cig} E(a, b)=\bigcup\left\{B\left(x, \frac{q(x)}{c}\right) \mid x \in E \backslash\{a, b\}\right\} \subset D .
$$


This definition is due to [V1, NV]. Bojarski proved that a bounded $b$-John domain satisfies the standard $(q, p)$-Poincare inequality [B, Chapter 6] with constant

$$
c=c(n, p, q) b^{n}|D|^{\frac{1}{n}+\frac{1}{q}-\frac{1}{p}} .
$$

Unbounded John domains are $\left(\frac{n p}{n-p}, p\right)$-Poincaré domains [H3, Corollary 4.6]

We need the following lemma due to Väisälä.

2.2. Lemma [V2]. Let $D$ be an unbounded b-John domain. There are bounded $b_{0}$-John domains $D_{i}$ such that $D_{i} \subset \bar{D}_{i} \subset D_{i+1}, i=1,2, \ldots$, and $D=$ $\bigcup_{i=1}^{\infty} D_{i}$.

Domains satisfying a quasihyperbolic boundary condition. The quasihyperbolic distance between points $x_{1}$ and $x_{2}$ in $D$ is given by

$$
k_{D}\left(x_{1}, x_{2}\right)=\inf _{\gamma} \int_{\gamma} \frac{d s}{d(x, \partial D)}
$$

where the infimum is taken over all rectifiable curves $\gamma$ joining $x_{1}$ and $x_{2}$ in $D$ [GP].

A domain $D$ satisfies a quasihyperbolic boundary condition, if there exists a point $x_{0} \in D$ and a constant $a>1$ such that

$$
k_{D}\left(x_{0}, x\right) \leq a \log \left(1+\frac{\left|x_{0}-x\right|}{\min \left\{d\left(x_{0}, \partial D\right), d(x, \partial D)\right\}}\right)
$$

for all $x \in D$.

John domains form a proper subclass of domains satisfying a quasihyperbolic boundary condition.

Whitney decomposition. By a Whitney decomposition of $D$ we mean a family $W$ of closed dyadic cubes, whose interiors are pairwise disjoint, and which satisfy

(1) $D=\bigcup_{Q \in W} Q$,

(2) $\operatorname{dia}(Q) \leq d(Q, \partial D) \leq 4 \operatorname{dia}(Q)$,

(3) $\frac{1}{4} \operatorname{dia}\left(Q_{2}\right) \leq \operatorname{dia}\left(Q_{1}\right) \leq 4 \operatorname{dia}\left(Q_{2}\right)$ when $Q_{1} \cap Q_{2} \neq \varnothing$.

Moreover, it follows from the construction in [S, Chapter VI], if $\sigma \in[1,5 / 4)$ is a fixed constant, then

$$
\sum_{Q \in W} \chi_{\sigma Q}(x) \leq 12^{n} \chi_{D}(x), \quad x \in \mathbb{R}^{n} .
$$

Cubes in $W$ are called Whitney cubes.

Sets $D_{i}, i=0,1, \ldots, k$, in $\mathbb{R}^{n}$ form a chain, abbreviated $C\left(D_{k}\right)=$ $\left(D_{0}, D_{1}, \ldots, D_{k}\right)$, if

$$
D_{i} \cap D_{j} \neq \varnothing \quad \text { if and only if }|i-j| \leq 1 .
$$

The next lemma relates the quasihyperbolic distance between points to the number of Whitney cubes in a chain joining these points. 
2.4. Lemma [H1, Proposition 6.1]. Fix $Q_{0} \in W$ and $x_{0} \in Q_{0}$. For each $Q \in W$ there is a chain $C(Q)=\left(Q_{0}, Q_{1}, \ldots, Q_{k}\right)$ of Whitney cubes joining $Q_{0}$ and $Q=Q_{k}$ such that for all $x \in \frac{9}{8} Q, k \leq c(n) k_{D}\left(x_{0}, x\right)+1$.

A Whitney cube \#-condition. Suppose that $D=\bigcup_{k=1}^{\infty} \bigcup_{j=1}^{N_{k}} Q_{j}^{k}$ and $|D|<\infty$; here the Whitney decomposition of $D$ (see [S, Chapter VI]) is arranged so that, for Whitney cubes $Q_{j}^{k}, \operatorname{dia}\left(Q_{j}^{k}\right)=|D|^{1 / n} 2^{-k}$ for $j=1, \ldots, N_{k}$. We say that $D$ satisfies a Whitney cube \#-condition, if there are constants $M<\infty$ and $\lambda \in(0, n)$ such that $N_{k} \leq M 2^{\lambda k}$ for $k=1,2, \ldots$.

Recall that if a domain $D$ satisfying a quasihyperbolic boundary condition has finite $n$-Lebesgue measure $|D|<\infty$, then $D$ is bounded [H3, Theorem 3.3].

\section{Proofs of THEOREMS AND EXAMPLES}

Proof of Theorem 1.3. (1) Suppose that $D$ is bounded. Let $W$ be a Whitney decomposition of $D$. Fix $Q_{0} \in W$ with $x_{0} \in Q_{0}$. By [H1, Lemma 2.3] it is enough to estimate

$$
\begin{aligned}
\int_{D}\left|u(x)-u_{\frac{9}{8} Q_{0}}\right|^{q} d x \leq & 2^{q} \sum_{Q \in W} \int_{\frac{9}{8} Q}\left|u(x)-u_{\frac{9}{8} Q}\right|^{q} d x \\
& +2^{q} \sum_{Q \in W} \int_{\frac{9}{8} Q}\left|u_{\frac{9}{8} Q}-u_{\frac{9}{8} Q_{0}}\right|^{q} d x .
\end{aligned}
$$

The ordinary $(q, p)$-Poincaré inequality holds in a cube, when $q \leq \frac{n p}{n-p}$ and $p<n$ [B, Chapter 6].

Hence using Whitney cube property (2) we obtain

$$
\begin{aligned}
& \sum_{Q \in W} \int_{\frac{q}{8} Q}\left|u(x)-u_{\frac{9}{8} Q}\right|^{q} d x \\
& \quad \leq c_{1}(n, p, q) \sum_{Q \in W}\left(|Q|^{\frac{1}{n}+\frac{1}{q}-\frac{1}{p}}\right)^{q}\left(\int_{\frac{q}{8} Q}|\nabla u(x)|^{p} d x\right)^{q / p} \\
& \quad \leq c_{2}(n, p, q) \sum_{Q \in W}\left(|Q|^{\frac{1}{n}+\frac{1}{q}-\frac{1}{p}-\frac{\delta}{n}}\right)^{q}\left(\int_{\frac{q}{8} Q}|\nabla u(x)|^{p} d(x, \partial D)^{\delta p} d x\right)^{q / p} \\
& \quad \leq c_{3}(n, p, q)|D|^{1+q\left(\frac{1-\delta}{n}-\frac{1}{p}\right)}\left(\int_{D}|\nabla u(x)|^{p} d(x, \partial D)^{\delta p} d x\right)^{q / p},
\end{aligned}
$$

since $\frac{q}{p} \geqslant 1, q \leq \frac{n p}{n-p(1-\delta)}$, and $p(1-\delta)<n$.

To estimate the sum

$$
\sum_{Q \in W} \int_{\frac{9}{8} Q}\left|u_{\frac{9}{8} Q}-u_{\frac{9}{8} Q_{0}}\right|^{q} d x
$$

fix $Q \in W$. We use the idea from [IN, Theorem 3]. According to [H1, Lemma 8.3] there is a cube $Q_{0} \in W$ such that each $Q \in W$ can be joined to $Q_{0}$ by a chain of cubes $Q_{j} \in W, j=0,1, \ldots, k, Q_{k}=Q$, such that

$$
Q_{l} \subset c_{4}(n) b Q_{j}
$$


for all $l \geq j$. Since we will rely on the triangle inequality,

$$
\left|u_{\frac{9}{8} Q}-u_{\frac{9}{8} Q_{0}}\right|^{q} \leq\left(\sum_{j=1}^{k}\left|u_{\frac{9}{8} Q_{j}}-u_{\frac{9}{8} Q_{j-1}}\right|\right)^{q},
$$

to achieve our estimate, we first provide an upper bound for each term on the right-hand side. The Whitney cube properties and the $(p, p)$-Poincaré inequality for cubes yield

$$
\begin{aligned}
\mid u_{\frac{9}{8} Q_{j}} & -\left.u_{\frac{9}{8} Q_{j-1}}\right|^{p}=\frac{1}{\left|\frac{9}{8} Q_{j} \cap \frac{9}{8} Q_{j-1}\right|} \int_{\frac{9}{8} Q_{j} \cap \frac{9}{8} Q_{j-1}}\left|u_{\frac{9}{8} Q_{j}}-u_{\frac{9}{8} Q_{j-1}}\right|^{p} d y \\
& \leq \frac{2^{p}}{\left|\frac{9}{8} Q_{j} \cap \frac{9}{8} Q_{j-1}\right|} \sum_{h=j-1}^{j} \int_{\frac{9}{8} Q_{h}}\left|u(y)-u_{\frac{9}{8} Q_{h}}\right|^{p} d y \\
& \leq c_{5}(n, p, \delta) \sum_{h=j-1}^{j}\left|Q_{h}\right|^{\frac{(1-\delta) p}{n}-1} \int_{\frac{9}{8} Q_{h}}|\nabla u(y)|^{p} d(y, \partial D)^{\delta p} d y .
\end{aligned}
$$

Thus using (3.3) we obtain

$$
\begin{aligned}
& \sum_{j=1}^{k}\left|u_{\frac{9}{8} Q_{j}}-u_{\frac{9}{8} Q_{j-1}}\right| \chi_{\frac{9}{8} Q_{k}}(x) \\
& \quad \leq c_{6}(n, p, \delta) \sum_{j=0}^{k}\left(\left|Q_{j}\right|^{\frac{(1-\delta) p}{n}-1} \int_{\frac{9}{8} Q_{j}}|\nabla u(y)|^{p} d(y, \partial D)^{\delta p} d y \chi_{c_{4} Q_{j}}(x)\right)^{1 / p} .
\end{aligned}
$$

The constants $c_{i}, i=7,8,9,10$, will depend at most on $n, p, q$, and $\delta$. Hence the above estimates [Bo, Lemma 3.3] and the inequality (2.3) imply

$$
\begin{aligned}
& \sum_{Q \in W} \int_{\frac{q}{8} Q}\left|u_{\frac{9}{8} Q}-u_{\frac{9}{8} Q_{0}}\right|^{q} d x \\
& \leq c_{7} \int_{\mathbb{R}^{n}}\left(\sum_{A \in C(Q)}\left[|A|^{\frac{(1-\delta) p}{n}-1} \int_{\frac{q}{8} A}|\nabla u(y)|^{p} d(y, \partial D)^{\delta p} d y \chi_{c_{4}(n) b A}(x)\right]^{1 / p}\right)^{q} d x \\
& \leq c_{8} b^{n q} \int_{\mathbb{R}^{n}}\left(\sum_{A \in W}\left[|A|^{\frac{(1-\delta) p}{n}-1} \int_{\frac{9}{8} A}|\nabla u(y)|^{p} d(y, \partial D)^{\delta p} d y \chi_{A}(x)\right]^{1 / p}\right)^{q} d x \\
& \leq c_{9} b^{n q} \sum_{A \in W}|A|^{\frac{q(1-\delta)}{n}-\frac{q}{p}}\left[\int_{\frac{9}{8} A}|\nabla u(y)|^{p} d(y, \partial D)^{\delta p} d y\right]^{q / p}\left[\int_{\mathbb{R}^{n}} \chi_{A}(x) d x\right] \\
& \leq c_{9} b^{n q} \sum_{A \in W}|A|^{\frac{q(1-\delta)}{n}-\frac{q}{p}+1}\left(\int_{\frac{9}{8} A}|\nabla u(y)|^{p} d(y, \partial D)^{\delta p} d y\right)^{q / p} \\
& \leq c_{10} b^{n q}|D|^{1+q\left(\frac{1-\delta}{n}-\frac{1}{p}\right)}\left(\int_{D}|\nabla u(y)|^{p} d(y, \partial D)^{\delta p} d y\right)^{q / p}
\end{aligned}
$$

where $p \leq q$ and $(1-\delta) \frac{1}{n}-\frac{1}{p}+\frac{1}{q} \geq 0$; here $p(1-\delta)<n$. 
Estimates (3.1), (3.2), and (3.6) together yield the desired inequality when $D$ is bounded.

(2) Suppose that $D$ is unbounded. By Lemma $2.2 D$ can be exhausted using bounded $b_{0}$-John domains $D_{i}$ such that $D_{i} \subset \bar{D}_{i} \subset D_{i+1}, i=1,2, \ldots$, and $D=\bigcup_{i=1}^{\infty} D_{i}$. The proof for Theorem 1.3 shows that each $D_{i}$ satisfies the improved Poincaré inequality with constant

$$
c\left(p, q, \delta, D_{i}\right)=b_{0}^{n}\left|D_{i}\right|^{\frac{1-\delta}{n}+\frac{1}{q}-\frac{1}{p}} .
$$

Applying a result on unions of Poincare domains, namely, Theorem 4.1 in $\S 4$, the proof for the unbounded case can be completed .

Proof of Theorem 1.4. The constants $c_{i}, i=1,2,3,4$, depend at most on $n, p, q, \delta$, and $D$. Let $W$ be a Whitney decomposition of $D$ and fix $Q_{0} \in W$ with $x_{0} \in Q_{0}$.

According to the proof of Theorem 1.3 (see (3.1) and (3.2)), we only need to estimate the sum

$$
\sum_{Q \in W} \int_{\frac{9}{8} Q}\left|u_{\frac{9}{8} Q}-u_{\frac{9}{8} Q_{0}}\right|^{q} d x .
$$

Fix $Q \in W$. By [H1, Lemma 7.13] there is a chain $C(Q)$ of Whitney cubes $Q_{j}, j=0,1, \ldots, k, Q_{k}=Q$, such that

$$
\operatorname{dia}\left(Q_{l}\right) \leq c_{1} \operatorname{dia}\left(Q_{j}\right)^{1 / a},
$$

$l \geq j$. Applying the method of [H1, Theorem 4.4] and using (3.4), (3.5), and Lemma 2.4 we obtain

$$
\begin{aligned}
\sum_{Q \in W} & \int_{\frac{9}{8} Q}\left|u_{\frac{9}{8} Q}-u_{\frac{9}{8} Q_{0}}\right|^{q} d x \\
\leq & c_{2} \sum_{Q \in W} \int_{\frac{9}{8} Q}\left(k_{D}\left(x_{0}, x\right)+1\right)^{q-1} d x \\
& \times \sum_{A \in C(Q)}\left(|A|^{\frac{p}{n}(1-\delta)-1} \int_{\frac{9}{8} A}|\nabla u(y)|^{p} d(y, \partial D)^{\delta p} d y\right)^{q / p} .
\end{aligned}
$$

Let $p(1-\delta)-n<0$. We utilize inequality (3.7),

$$
\begin{aligned}
& \sum_{Q \in W} \int_{\frac{q}{8} Q}\left(k_{D}\left(x_{0}, x\right)+1\right)^{q-1} d x \\
& \quad \times \sum_{A \in C(Q)}\left(|A|^{\frac{p}{n}(1-\delta)-1} \int_{\frac{q}{8} Q}|\nabla u(y)|^{p} d(y, \partial D)^{\delta p} d y\right)^{q / p} \\
& \leq c_{3} \sum_{Q \in W} \int_{\frac{q}{8} Q}\left(k_{D}\left(x_{0}, x\right)+1\right)^{q-1}|Q|^{q a\left((1-\delta) \frac{1}{n}-\frac{1}{p}\right)} d x \\
& \quad \times \sum_{A \in C(Q)}\left(\int_{\frac{\frac{9}{8}}{8} A}|\nabla u(y)|^{p} d(y, \partial D)^{\delta p} d y\right)^{q / p} .
\end{aligned}
$$


Now [H1, Theorem 7.7] and [SS, Corollary 1] yield

$$
\begin{aligned}
& \sum_{Q \in W} \int_{\frac{q}{8} Q}\left(k_{D}\left(x_{0}, x\right)+1\right)^{q-1}|Q|^{q a\left((1-\delta) \frac{1}{n}-\frac{1}{p}\right)} d x \\
& \leq c_{4} \sum_{j=1}^{\infty} j^{q-1} 2^{\lambda j} 2^{-n j} 2^{-\frac{q a}{p}((1-\delta) p-n) j}<\infty,
\end{aligned}
$$

if

$$
n-\lambda+\frac{q a}{p}((1-\delta) p-n)>0
$$

here $\lambda<n$ is a Whitney cube \#-constant. Combining inequalities (3.1), (3.2), and (3.8)-(3.10) we find that there is a constant $c<\infty$ such that

$$
\left\|u(x)-u_{D}\right\|_{L^{q}(D)} \leq c\left\|\nabla u(x) d(x, \partial D)^{\delta}\right\|_{L^{p}(D)},
$$

whenever $\frac{1}{q}-\frac{1}{p}+\frac{1-\delta}{n} \geq 0$ and $\frac{n-\lambda}{q a}-\frac{1}{p}+\frac{1-\delta}{n} \geq 0$, where $p(1-\delta)<n$.

3.11. Remarks. (1) The following example shows that even in the case of John domains one must require $\delta \leq 1$.

We use the following notation for the upper half of the disk $B^{2}(0, r)$ :

$B_{+}(r)=B^{2}(0, r) \cap\left\{\left(x_{1}, x_{2}\right) \mid x_{2}>0\right\}, r>0$

Our domain will be a ball with a slit removed. In particular, we examine

$$
D=B^{2}(0,4) \backslash\left\{\left(x_{1}, 0\right) \| x_{1} \mid<3\right\} \text {. }
$$

Define the following subsets of $D$ :

$$
\begin{aligned}
& D_{1}=B^{2}(0,4) \cap\left\{\left(x_{1}, x_{2}\right) \mid 0<x_{2}<x_{1}-2\right\}, \\
& D_{-1}=B^{2}(0,4) \cap\left\{\left(x_{1}, x_{2}\right) \mid 0<x_{2}<-x_{1}-2\right\}, \\
& D_{2}=B_{+}(4) \backslash\left(B_{+}(2) \cup D_{1} \cup D_{-1}\right) .
\end{aligned}
$$

We construct a symmetric function $u(x)$ in $D$ as follows. Let

$$
u(x)= \begin{cases}|x|^{-\frac{2}{p}} & \text { on } B_{+}(1), \\ -2|x|+3 & \text { on } B_{+}(2) \backslash B_{+}(1), \\ -1 & \text { on } D_{2}, \\ x_{2} /\left(x_{1}-2\right) & \text { on } D_{1}, \\ -x_{2} /\left(x_{1}+2\right) & \text { on } D_{-1}, \\ 0 & \text { on }\left\{\left(x_{1}, 0\right)|3 \leq| x_{1} \mid<4\right\}\end{cases}
$$

and set $u\left(x_{1},-x_{2}\right)=-u\left(x_{1}, x_{2}\right)$.

This function $u$ shows that $D$ does not satisfy the improved Poincare inequality (1.2), if $\delta>1$.

(2) The following example shows that $\delta$ is strictly less than 1 when $D$ is not a John domain but satisfies a quasihyperbolic boundary condition.

Let $G_{0}$ be the open square bounded by the lines

$$
x_{1}=0, \quad x_{2}=0, \quad x_{1}=1, \quad x_{2}=-1,
$$

and for $j=1,2, \ldots$ let $G_{j}$ be the open triangle bounded by

$$
x_{1}=2^{-2 j}, \quad x_{2}=2^{-2 j}-2^{-2 b j}, \quad x_{1}+x_{2}=2^{-2 j}-2^{-2 b j},
$$

where $b \geq 2$ is a constant. Denote by $\widehat{G}$ the reflection of the domain $\bigcup_{j=0}^{\infty} G_{j}$ with respect to the line $x_{2}=-\frac{1}{2}$. Set

$$
G=\bigcup_{j=1}^{\infty} G_{j} \cup \widehat{G} .
$$


Let $T: \mathbb{R}^{2} \rightarrow \mathbb{R}^{2}$ be the translation $T\left(x_{1}, x_{2}\right)=\left(x_{1}, x_{2}+\frac{1}{2}\right)$. Set $D=T(G)$. $D$ satisfies a quasihyperbolic boundary condition with $a=36 b$.

Let $G_{j}^{1}$ be the open set bounded by the lines $x_{1}=2^{-2 j}, x_{2}=2^{-2 j}-2^{-2 b j}$, $x_{2}=2^{-2 b j}, x_{1}+x_{2}=2^{-2 j}-2^{-2 b j}$. Let $\widehat{G}_{j}^{1}$ be the image of $G_{j}^{1}$ under reflection across the line $x_{2}=-\frac{1}{2}$. Set $T\left(G_{j}^{1}\right)=D_{j}^{1}$ and $T\left(\widehat{G}_{j}^{1}\right)=\widehat{D}_{j}^{1}$.

Choose a piecewise linear continuous function $u: D \rightarrow \mathbb{R}$ such that

$$
u(x)= \begin{cases}2^{4 j / q} & \text { in } D_{j}^{1}, j=1,2, \ldots, \\ 0 & \text { in }\left\{\left(x_{1}, x_{2}\right) \mid x_{1} \in(0,1), x_{2} \in\left(-\frac{1}{2}, \frac{1}{2}\right)\right\}, \\ -2^{4 j / q} & \text { in } \widehat{D}_{j}^{1}, j=1,2, \ldots\end{cases}
$$

We conclude that $u$ does not satisfy the improved Poincaré inequality (1.2) for any $p$.

(3) The upper bound for $q$ in Theorem 1.4, when $D$ satisfies a quasihyperbolic boundary condition and $p(1-\delta)<n$, is essentially sharp, $q \leq \frac{(n-\lambda) n p}{a(n-p)}$ (see the case $\delta=0$ in [H3, Example 3.7]).

(4) There are domains which are $(p, p)$-Poincare domains for each $p \geq 1$, but which do not satisfy the improved Poincaré inequality (1.2) for any $\delta>0$. We construct such a "rooms and passages" domain. Let

$$
G_{1}=\bigcup_{i=1}^{\infty}\left(D_{2 i-1} \cup P_{2 i}\right)
$$

where the sets $D_{2 i-1}$ and $P_{2 i}, i=1,2, \ldots$, are defined as follows: Let $\left(h_{i}\right)$ and $\left(\eta_{2 i}\right)$ be sequences, where $h_{i}=M^{-i}, M>1$, and $\eta_{2 i}=b M^{-2 a i}, b>0$, $a>1$. Write $\sum_{i=1}^{k} h_{i}=d_{k}, k=1,2, \ldots$ Define

$$
\begin{gathered}
D_{2 i-1}=\left(d_{2 i-1}-h_{2 i-1}, d_{2 i-1}\right) \times\left(-\frac{1}{2} h_{2 i-1}, \frac{1}{2} h_{2 i-1}\right)^{n-1}, \\
P_{2 i}=\left[d_{2 i-1}, d_{2 i-1}+h_{2 i}\right] \times\left(-\frac{1}{2} \eta_{2 i}, \frac{1}{2} \eta_{2 i}\right)^{n-1},
\end{gathered}
$$

$i=1,2, \ldots$. Define $G=G_{1} \cup G_{2} \cup G_{3}$, where $G_{2}$ is the reflection of $G_{1}$ with the hyperplane $x_{1}=0$ and $G_{3}=\left(-h_{1} / 2, h_{1} / 2\right)^{n}$. Let $\left(u_{k}\right), k=1,3,5, \ldots$, be a sequence of piecewise linear continuous functions which satisfy

$$
u_{k}(x)= \begin{cases}h_{k}^{-(n / p)} & \text { in } D_{k}, \\ 0 & \text { in } G_{1} \backslash\left\{P_{k-1} \cup D_{k} \cup P_{k+1}\right\} .\end{cases}
$$

Extend the functions $u_{k}$ to $G$ as odd functions of $x_{1}$. The constans $c_{1}$ and $c_{2}$ below depend only on $a, b, n$, and $M$. We can compute that

$$
\int_{G}\left|u(x)_{2 i-1}\right|^{p} d x \geq c_{1}
$$

and

$$
\int_{G}\left|\nabla u(x)_{2 i-1}\right|^{p} d(x, \partial D)^{\delta p} d x \leq c_{2} M^{-2 i((n-1)(a-1)-p+a \delta p)} \rightarrow 0,
$$

as $i \rightarrow \infty$. Thus $G$ does not satisfy the improved Poincaré inequality, if $\delta>\frac{1}{a}\left(1-\frac{(n-1)(a-1)}{p}\right)=\delta_{0}$. Here $\delta_{0} \in(0,1)$.

On the other hand by [H1, Remark 5.9] $G \in \mathscr{P}(p, p)$ if and only if $p \geq$ $(n-1)(a-1)$. Note that notation there does not coincide with the notation here. 
There are also star-shaped domains which do not satisfy the improved Poincaré inequality (1.2) for any $\delta>0$. Recall that a star-shaped domain with respect to a point is a $(p, p)$-Poincare domain for each $p \geq 1[\mathrm{H} 1$, Theorem 3.1]. The following domain is from [BS, 4(1)]. Let $D=\left\{\left(x_{1}, x_{2}\right) \mid 0<\right.$ $\left.x_{1}<1,0<x_{2}<x_{1}^{1 / \alpha}\right\}, 0<\alpha \leq 1$, and suppose that $\delta>\alpha$. Define $u\left(x_{1}, x_{2}\right)=\left|\left(x_{1}, x_{2}\right)\right|^{-\frac{1+1 / \alpha}{p}}$. Then $u_{D}<\infty$. The function $v(x)=u(x)-u_{D}$, $x \in D$, does not satisfy (1.1), whenever $\delta>\alpha$.

\section{FURTHER REMARKS}

We have the following theorem for unbounded domains. Theorem 4.1 is a generalization of the case $\delta=0$ in [H3, Theorem 4.1], but the proof for $\delta \in[0,1]$ requires only minor modifications .

4.1. Theorem. Let $\delta \in[0,1]$ be a fixed number. Suppose that $D$ in $\mathbb{R}^{n}$ is an unbounded domain such that $D=\bigcup_{i=1}^{\infty} D_{i}$, where the bounded domains $D_{i}$ satisfy the improved $\left(\frac{n p}{n-p(1-\delta)}, p\right)$-Poincare inequality (1.2) with constants $c\left(n, p, \delta, D_{i}\right) \leq c_{0}$ for some constant $c_{0}<\infty$, and $D_{i} \subset \bar{D}_{i} \subset D_{i+1}, i=$ $1,2, \ldots$, and $\left|D_{1}\right|>0$. Then $D \in \mathscr{P}(q, p, \delta)$ where $p \leq q=\frac{n p}{n-p(1-\delta)}$ and $(1-\delta) p<n$.

Theorem 1.3 implies the following interesting corollary.

4.2. Corollary. Suppose that $D$ is an unbounded b-John domain. There is a constant $c<\infty$ such that

$$
\inf _{a \in R}\|u(x)-a\|_{L^{p}(D)} \leq c\|\nabla u(x) d(x, \partial D)\|_{L^{p}(D)}
$$

holds whenever $u \in L_{\text {loc }}^{1}(D), \nabla u(x) d(x, \partial D) \in L^{p}(D)$, and $1 \leq p<n$.

Edmunds and Opic have studied examples of domains satisfying (4.3), when $n=1$ [EO, Example 5.4].

\section{ACKNOWLEDGMENTS}

I would like to thank W. Smith for bringing this problem to my attention, J. Väisälä for the use of his unpublished result Lemma 2.2, and S. Staples for carefully reading the manuscript.

\section{REFERENCES}

[BS] H. B. Boas and E. J. Straube, Integral inequalities of Hardy and Poincaré type, Proc. Amer. Math. Soc. 103 (1988), 172-176.

[B] B. Bojarski, Remarks on Sobolev imbedding inequalities, Complex Analysis (Joensuu 1987), Lecture Notes in Math., vol. 1351, Springer-Verlag, Berlin and Heidelberg, 1988, pp. 52-68.

[Bo] J. Boman, $L_{p}$-estimates for very strongly elliptic systems, Department of Mathematics, University of Stockholm, Sweden, Report no. 29, 1982.

[EO] D. E. Edmunds and B. Opic, Weighted Poincaré and Friedrichs inequalities, J. London Math. Soc. 47 (1993), 79-96.

[GP] F. W. Gehring and B. P. Palka, Quasiconformally homogeneous domains, J. Analyse Math. 30 (1976), 172-199.

[H1] R. Hurri, Poincaré domains in $\mathbb{R}^{n}$, Ann. Acad. Sci. Fenn. Ser. A I Math. Dissertationes 71 (1988), 1-41. 
[H2] The weighted Poincaré inequalities, Math. Scand. 67 (1990), 145-160.

[H3] R. Hurri-Syrjänen, Unbounded Poincaré domains, Ann. Acad. Sci. Fenn. Ser. A I Math. 17 (1992), 409-423.

[IN] T. Iwaniec and C. A. Nolder, Hardy-Littlewood inequality for quasiregular mappings in certain domains in $\mathbb{R}^{n}$, Ann. Acad. Sci. Fenn. Ser. A I Math. 10 (1985), 267-282.

[K] A. Kufner, Weighted Sobolev spaces, Wiley, New York, 1985.

[M] V. G. Maz'ya, Sobolev spaces, Springer-Verlag, Berlin, Heidelberg, New York, and Tokyo, 1985.

[NV] R. Näkki and J. Väisälä, John disks, Exposition. Math. 9 (1991), 3-43.

[S] E. M. Stein, Singular integrals and differentiability properties of functions, Princeton Univ. Press, Princeton, NJ, 1970.

[SS] W. Smith and D. Stegenga, Exponential integrability of the quasi-hyperbolic metric on Hölder domains, Ann. Acad. Sci. Fenn. Ser. A I Math. 16 (1991), 344-359.

[V1] J. Väisälä, Quasiconformal maps of cylindrical domains, Acta Math. 162 (1989), 201-225.

[V2] _ Exhaustions of John domains, Ann. Acad. Sci. Fenn. Ser. A I Math. (to appear).

DePartment of Mathematics, UNiversity of JyVäskylä, SF-40351 JyVÄSKYlä, Finland E-mail address: hurri@f injyu.bitnet

Current address: Department of Mathematics, The University of Texas at Austin, Austin, Texas 78712

E-mail address: syrjanen@math.utexas.edu 